\title{
Developing Crew Allocation System for the Precast Industry Using Genetic Algorithms
}

\author{
Ammar Al-Bazi \\ Centre for Construction Innovation Research (CCIR), School of Science and \\ Engineering (SSE), Teesside University, UK \\ a.f.albazi@tees.ac.uk \\ $\&$ \\ Nashwan Dawood \\ Centre for Construction Innovation Research (CCIR), School of Science and \\ Engineering (SSE), Teesside University, UK \\ n.n.Dawood@tees.ac.uk
}

\begin{abstract}
:
The Precast Concrete Industry $(P C l)$ is one of the major contributors to the national economy and can be categorised as labor-intensive industry. It is currently experiencing shortcomings in terms of delivery products at a competitive cost and time. This is mainly due to the inefficiencies associate with planning and scheduling of skilled operators within crews configurations. This paper presents a new strategy for efficient allocation of crews of workers in the precast concrete industry using Genetic Algorithms-based simulation modeling. The aim of this study is to develop crew allocation system that can efficiently allocate possible crews of workers to precast concrete labor-intensive repetitive processes. Genetic Algorithms (GAs) have been developed to solve this type of problems. Process mapping methodologies were used to identify and document the processes involved in producing precast components. Then process simulation was
\end{abstract}




\section{Al-Bazi \& Dawood}

used to model and simulate all these processes and GAs were tailored to be embedded with the simulation model for a better search of promising solutions. GAs operators were designed to suite this type of allocation problem. "Class Interval" selection strategy was developed to give a greater opportunity for the promising chromosomes to be chosen for further investigation. Dynamic crossover and mutation operators were developed to add more randomness to the search mechanism. The results showed that adopting different combinations of crews of workers had a substantial impact on reducing the process throughput time, minimizing resources cost, and achieving the required operatives utilisation.

\section{Introduction}

'Crew allocation' can be defined as the process of assigning crews of workers or operatives to processes to ensure that they are used in an optimal way. This problem becomes more challenging when it involves a complex system that shares limited crews. Crew allocation can be a very complex problem when same operatives are required to be involved in concurrent or simultaneous processes. The optimal allocation of crews should satisfy minimum clashes or overlaps between different crew's operatives and this should subsequently minimize production costs and idle time.

Two difficulties can arise in solving this problem: the combinatorial explosion of alternative crews normally leads to NP-hard optimization problems and the lack of analytical expressions relating performance functions to solutions usually results in noisy estimates of the performances (Shi 2000).

There is a lack of applying appropriate techniques that advise on optimal allocation of crews of workers in the precast industry. It is crucial to plan and manage operatives allocation to ensure the optimal choice of crews and effective utilization of each crew member have been achieved, Zhang et. al., (2004). 


\section{Al-Bazi \& Dawood}

As one of sophisticated techniques that have been used in solving these kinds of problems, GAs have been applied to many different optimization problems such as the optimization of traffic control signals (Teklu et. al., 2007), electrical transmission towers (Mathakari et. al., 2007), (Cheng et. al., 2009), highway alignment optimization (Kang et. al., 2009), and large structures (Adeli and Kumar, 1995a\&b; Sarma and Adeli, 2000a\&b, 2001), as well as short-term urban traffic flow forecasting (Vlahogianni et. al., 2007), pattern recognition (Lee et al., 2007), classification of radar targets (Carro-Calvo et. al., 2009), structural control (Jiang and Adeli,2008), machine learning (Hung and Adeli, 1994), and robotics (Smith et. al., 2007). They are one of the most promising meta-heuristics algorithms (Alcaraz et. al., 2001). GA technique is defined as a computational model simulating the process of genetic selection and natural elimination in biological evolution, (Adeli and Cheng, 1994a\&b; Kumar et. al., 2009). In recent years, various approaches to construction simulation have been advanced such as object identification and tracking (Chi et. al., 2009) and high speed animation (Rekapalli et. al., 2009). In this work, GA is designed to be coupled with process-simulation model for further guidance of the simulation model toward choosing the best processing crew among sets of possible alternatives. The reason behind choosing GA is its ability to intelligently search through a solution space for optimal or near optimal solutions. In the crew allocation problem under study, an intelligent searching algorithm is developed to investigate a large pool of crews, to decide which set of crews can be allocated to processes in order to minimize labor costs.

In this paper, the developed crew allocation system dubbed "SIM_Crew" was designed to efficiently allocate crews of workers to the precast industry. In such an industry, there is a high demand for skilled workforce and skilled operatives costs are relatively high. A "sleeper" precast manufacturing system was considered as a test of the proposed system. A proper allocation system will thus ensure minimum throughput time, production costs, and maximum utilization of resources. A 'sleeper' precast component is used as a base for rail tracks. 'Sleepers' are precast 


\section{Al-Bazi \& Dawood}

components generally laid transverse to the rails, on which the rails are supported and fixed, to transfer the loads from rails to the ballast and sub grade below, and to hold the rails to the correct gauge.

The reminder of the paper discusses the research problem, previous theories concerning crew planning and scheduling techniques, system architecture of the crew allocation system, process mapping methodologies, simulation and optimization modeling and finally a real life case study to validate the model.

\section{Research Problem}

Due to the fact that the parallel and sequential nature of any labor-driven system is pre-specified, the labor shared between similar or different tasks is generally allocated according to the workflow. However, idle times are still not carefully considered and the overall throughput time or total costs may be longer or higher than expected. In fact, this problem becomes more apparent when there is significant idle time for a process due to sharing operatives.

Due to the fact that the precast operations are labor-intensive and the required workforce involved in such industry is highly skilled and costly, optimization of crew allocation in the precast manufacturing system is needed in order to minimize idle times caused by sharing operatives.

Each production process has a certain pool of crews that can possibly perform the work for each of the parallel activities. Each crew has a different set of workers "different crew formation" with a certain process time. Sharing available operatives in different crews will eventually cause delays.

'Parallel-Repetitive Similar Processes' is a realistic example of a resource sharing case. All production processes in such a layout have a similar sequence, and the same materials are processed by similar parallel repetitive processes in order to produce the same or different 


\section{Al-Bazi \& Dawood}

products in a short cycle time. This kind of production layout can be seen in a job-shop environment such as seen in the precast concrete industry.

The aim of "SIM_Crew" is to achieve the best allocation of crews to carry out parallel or sequential processes for any labor-driven facility. The optimal crew allocation will eventually reduce the clashes between any shared resources, and subsequently lead to minimizing both operatives costs and process idle times. The system architecture of the crew allocation system will be described in the next section.

\section{Literature Review}

Crew planning and scheduling using simulation modeling and optimization technology have been applied in construction and precast industries. The layout of the operations (parallel/series) and repeatability of operations involved was crucial when considered in such planning and scheduling analysis. In this context, the literature review is categorized into two groups:

\section{Repetitive Operations}

In this type of layout, crews are often required to repeat the same work in various locations of the enterprise, moving from one location to another. Many available crew planning and scheduling systems for repetitive operations have been discussed in the work of Nassar (2005) who presented a model that uses a spreadsheet based GA models to optimally assign resources to repetitive activities of construction projects. Ipsilandis (2006) presented a linear programming parametric model for supporting construction managers decisions, exploring the multi-objective nature of decision making in repetitive construction projects. The project consisted of six repetitive units each having six discrete activities repeated in each unit. Lu et. al., (2008) presented a computer system dubbed "simplified simulation-based scheduling (S3)" to solve the problem of skilled laborer scheduling in a multi-site, multi-project context. The model consists of an optimizer using the Particle Swarm Optimizer (PSO) as a stochastic search strategy on a 


\section{Al-Bazi \& Dawood}

population of individuals, each representing a possible solution to the problem. Dawood et. al., (2007), developed a generic simulation model depicting the operational processes of the precast concrete production system. Results showed that the smaller the number of human resource available, the longer will be the lead time and the production costs will be lower. The greater the number of resources, the shorter the lead time, however, production costs will be greater.

\section{Non-Repetitive Operations}

In non-repetitive processes/ activities, crews are often required to carry out non-similar works in various locations of the project / job-shop. Many available scheduling methods for repetitive construction projects have been presented in the work of Moselhi et. al., (2007), who proposed a new methodology utilizing combined GAs and spatial technologies for optimization of crew formations for earthmoving operations. The designed model is useful for planning, tracking and control of earthmoving operations. Watkins et. al., (2007), used agent-based modeling methods simulating space congestion on a construction site to explore the impact of individual interactions on productivity and labor flow. Li et. al., (1998), presented a methodology for optimizing labor and equipment assignment for excavation and earthwork tasks using the Genetic Algorithm. A number of modifications of the basic GAs were achieved to improve the capacity of the algorithms. Zhang et. al., (2004), developed an optimization methodology which integrates discrete-event simulation with an heuristic algorithm to optimize dynamic resource allocation for construction scheduling. Senouci et. al., (2001), presented an optimization formulation for project scheduling problems with the objective of minimizing the total construction cost. The developed model considers project scheduling characteristics generally ignored in Adeli and Karim (1997) including precedence relationships, multiple crew-strategies, and time-cost tradeoff, they addressed resource levelling and resource-constrained scheduling of non-repetitive activities performed simultaneously. Marzouk et. al., (2004), provides a multi-objective 


\section{Al-Bazi \& Dawood}

optimization tool to optimize earthmoving operations using computer simulation and genetic algorithms. The optimization is aimed at minimizing the time and cost of earthmoving operations. Marzouk et. al., (2007), presented a special purpose simulation model to capture the uncertainty associated with bridge construction and results of the model showed that increasing the number of labor crews shortens the fabrication duration. The above literature study presented many methodologies and tools used to solve crew allocation problems in both repetitive and nonrepetitive operations layout in construction and precast industries, most of which have focused on minimizing costs rather than considering the effects of resources utilization on system performance. Some studies demonstrated the simulation of repetitive processes but the detailed crew formations of each process were not taken into account. Many systems have not used any optimization modules for a crew allocation; only running scenarios for improvement were adopted in such systems. In addition, available performance keys such as costs and utilization were not calculated to show the effects on system performance. Crew planning and scheduling systems are incapable of determining worker utilization as they can only produce crew utilization, so there is a pressing need for advanced systems that can address the utilization issue. The proposed system presented in this paper, more attention has been given to the worker utilization factors as they impact labor allocation cost. The next section will describe the research problem in more detail.

\section{Architecture of Crew Allocation System}

The purpose of allocating crews is to place the most suitable team of workers for each activity at minimum allocation cost and best possible performance. Therefore, as a fundamental requirement, crews should be allocated to new production processes once they are freed from previous processes. In addition, the performance of alternative feasible crews should be evaluated in terms of allocation cost and effciency, so that the most efficient one with the minimum cost 
and less interruption will be selected for implementation. To meet the above allocation process objective, a GA based simulation model is proposed, as shown in figure 1 .

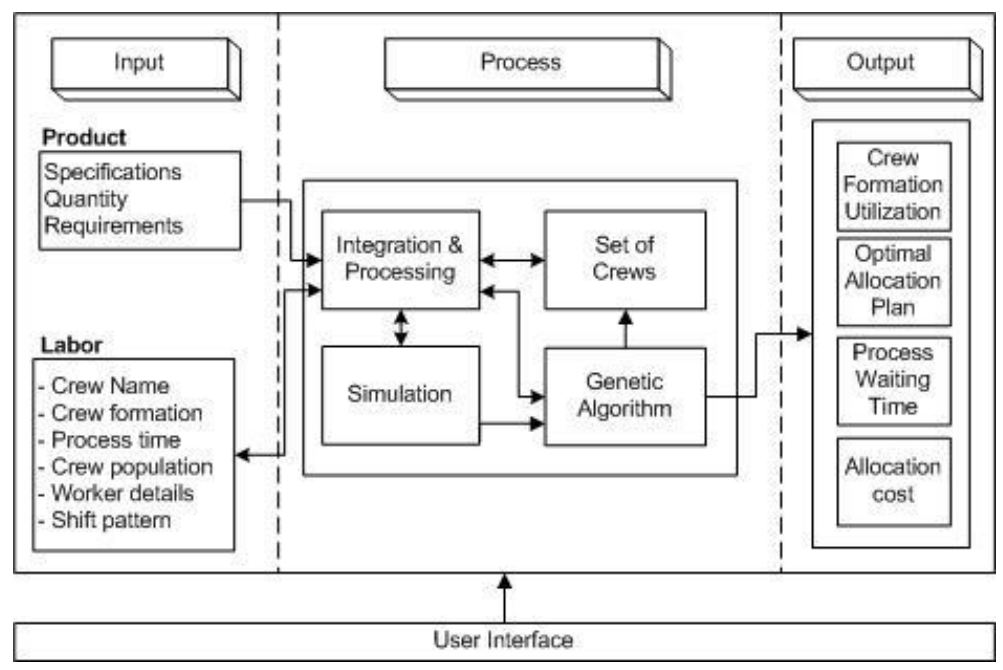

Figure 1: "SIM_Crew" Architecture

As shown in figure 1, the core of "SIM_Crew" is the simulation model which is integrated with databases through integration, and a processing module developed by writing special codes to enable such integration. The optimization engine is designed to be embedded in the simulation model; solutions are suggested from this engine to be evaluated by the simulation model, and all results are then stored in the database. During one allocation iteration, the simulation executes allocation plans: each of them has sets of crews to be allocated to the production processes, while GA evaluates the performance of the resultant allocation, and based on this, adjusts the decision variables and selects the most promising once. The main functionality of the optimization engine is to propose and manipulate solutions to be tested by the simulation model for a better performance. A number of key performance indicators such as total resource allocation cost, crew formation utilization, and other fixed resources utilization were used to evaluate the outputs of the model. An interface mechanism is designed to include size of population, crossover and mutation strategies, and other entry requirements. As shown in figure 1, the allocation process is an iterative procedure of progressive improvement. 


\section{Process Mapping, Simulation and Optimization Modeling}

\subsection{Process Mapping}

All production processes at two production sections are mapped to identify inputs, resources, constraints affecting each process, and the resulting outputs of each production process. This type of visual modeling is important to identify the relationships among the production process and the logic of each process. In order to have a sound understanding of the production life cycle at each production section, all production processes were studied and mapped. Figure 2 shows IDEF0 diagrams of one of the production lines (four production lines were considered in this study, each of them with a similar production sequence).



Figure 2: sleeper manufacturing process mapping diagram

The process flow starts from the mold setup process where inputs of this process are defined. The restrictions imposed on this process are mold and labor availability. The resources required to carry out the setup process include: an air compressor, a mold, laborers. The second process is to 


\section{Al-Bazi \& Dawood}

place strands inside the mold so that the sleepers can be reinforced, as a third process may require different numbers of strands depending on the type of products (sleepers). The casting process is then applied to fill the mold with the required amount of concrete and fourthly, the curing process uses a steaming system to cure the cased sleepers. The curing area capacity and availability of plastic sheets are the main restrictions on the curing process. Demolding follows the cutting process, involving cutting off the strands and then demolding sleepers with a special machine. The finishing of the sleepers is the last process which comprises placing plastic, rubber, and clips on each sleeper.

All inputs, outputs, constraints, and resources regarding each process are defined to give a clear idea about the core of each process and how these processes can be related to each other so that an accurate simulation model can be developed. The following section details the simulation model developed in this research project.

\subsection{Simulation Modeling}

The ability of simulation to model physical processes on a computer, incorporating the uncertainties that are inherent in all real systems, provides an enormous advantage for analysis in situations that are too complex to model mathematically (Glover, et. al., 1999). As one of the common methodologies used to simulate real life systems, Discrete Event Simulation (DES) is used to simulate production processes of the manufacturing system being investigated, as it is a powerful methodology used to model many real systems as they progress through time. The primary objective of the developed simulation model is to analyze "what-if" scenarios and thereby evaluate the allocation of each set of crews of workers to production processes for any manufacturing system. All inputs, process, and output of the simulation model are mapped in as introduced in the previous section and more details are explained in the next sections. 


\section{Al-Bazi \& Dawood}

\section{- Inputs}

Demands and orders specifications have been identified which will be produced in each production line. Information about orders includes the type of product, quantity needed, the amount of materials required for each order and resource needed. All production information is stored in an Excel spreadsheet to be integrated with ARENA using the Data Access Object "DAO". All other labor information is stored in Access databases. The skills needed for each crew for each process have been determined by and possible crews formation.

A crew alternative list for each process has been provided by the production manager (according to his experience). All sets of possible crews are accommodated into the Access database for further use. See figure 3:

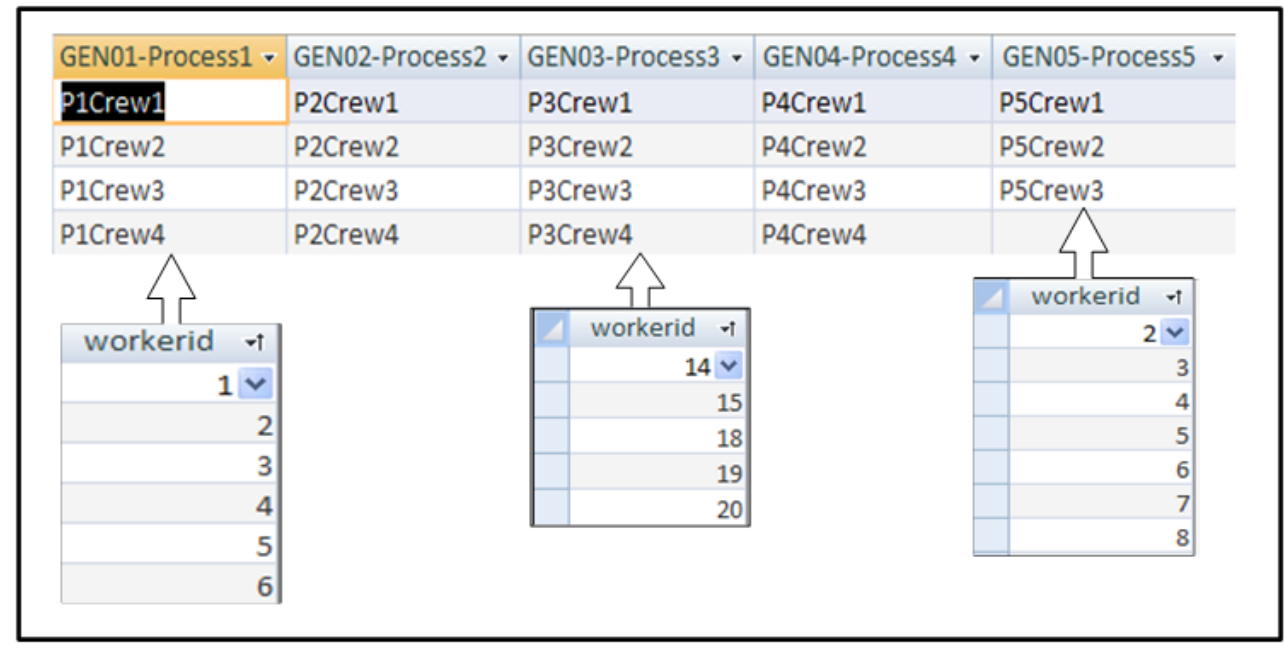

Figure 3: shows the crew population pool and some crew formation

In figure 3, each crew of workers involves a set of workers that are qualified to carry out a process. The workers were coded according to their numbers and all related information saved in the workers' pool. The qualification of each worker as well as hourly costs were saved in the workers' pool. 


\section{Al-Bazi \& Dawood}

\section{- Process}

This includes all the simulated production processes which are introduced in section 5.1.

\section{- Outputs}

After running the simulation model the outputs are stored in Excel files for further analysis. Some of the results are stored in the Access database to check for chromosome duplication. A large number of text files are created to validate the working of the optimization engine operators. The parameters evaluated include total resource allocation cost, and crew formation utilization.

\section{Assumption of the Simulation Model}

A number of assumptions are used when constructing the simulation model, the purpose of these assumptions was to simplify the development of the simulation model.

1. Each crew member is intensively involved in carrying out the production process. All members work together to finalise the process.

2. To begin a production process, all crew members should be available and should be released at once when that the process is complete.

3. The model is developed based on 24 hour working.

4. Only direct costs are considered in this model

\subsection{Optimization Using Genetic Algorithms}

The previous section has introduced the simulation model and the objective of this section is to discuss the optimization technology used in this research and the integration with the simulation model.

The benefit of using GA is the possibility to search for an optimum or near-optimum allocation of a crew of workers to each production process. This type of allocation optimizes both cost and 
production, under various constraint scenarios related to the availability of workers within a crew.

Figure 4 shows how GA is used to optimize the crew allocation process:

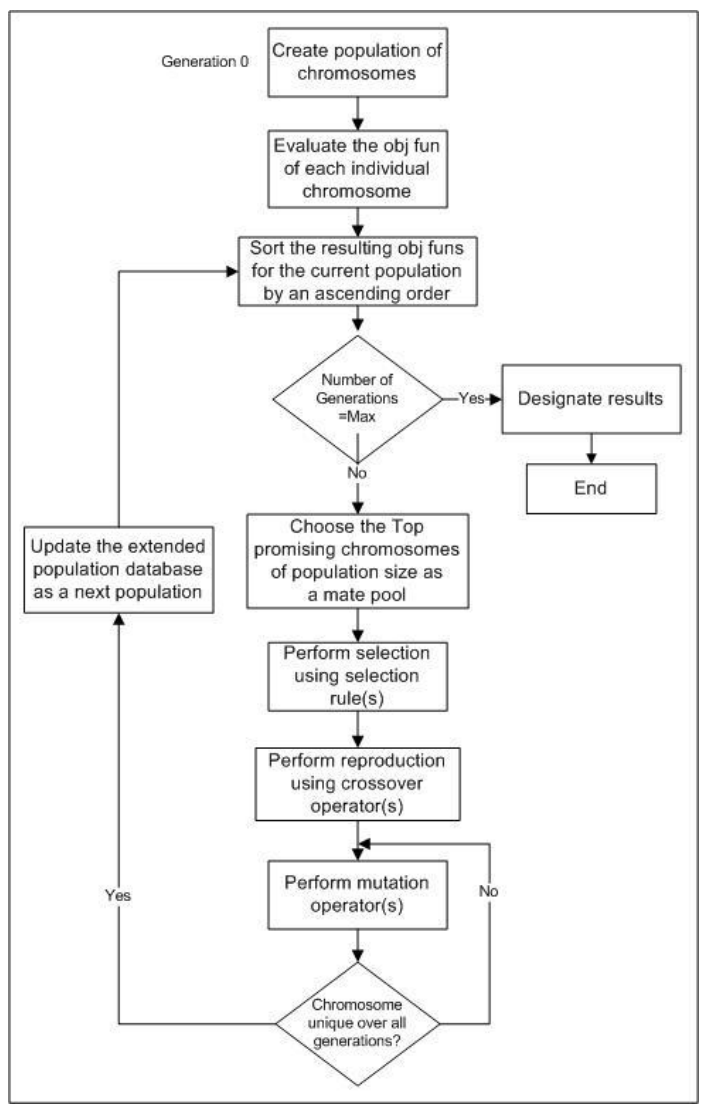

Figure 4: Modified Genetic Algorithms Flowchart

The initial population of chromosomes, each chromosome consisting of a set of crews to be assigned to processes generates random sets of crews using a Monte Carlo sampling technique. The most promising chromosomes should always be kept in the top list for easier access and further selection. For this purpose, the SQL query is set to always select the most promising chromosomes of a population size. Each pair of chromosomes is then selected to be prepared for further crossover and mutation purposes. An un-repeatability condition is imposed to avoid any repetition which might occur by producing two similar chromosomes. This process continues until the condition regarding the number of generations is satisfied. After each generation, the population is added to the previous generation's population to produce an augmented population pool. A sorting procedure is then applied on the augmented population pool. 


\section{Al-Bazi \& Dawood}

The output of the optimization process represents the possible 'set of crews' that can be allocated to each production process. The decision variables are stored in a row vector (string) called a chromosome, which has a number of elements (genes) representing the number of variables equal to the number of sets of crews available and possible to allocate. The chromosome was specifically designed to resolve this type of problem as demonstrated in figure 5 .

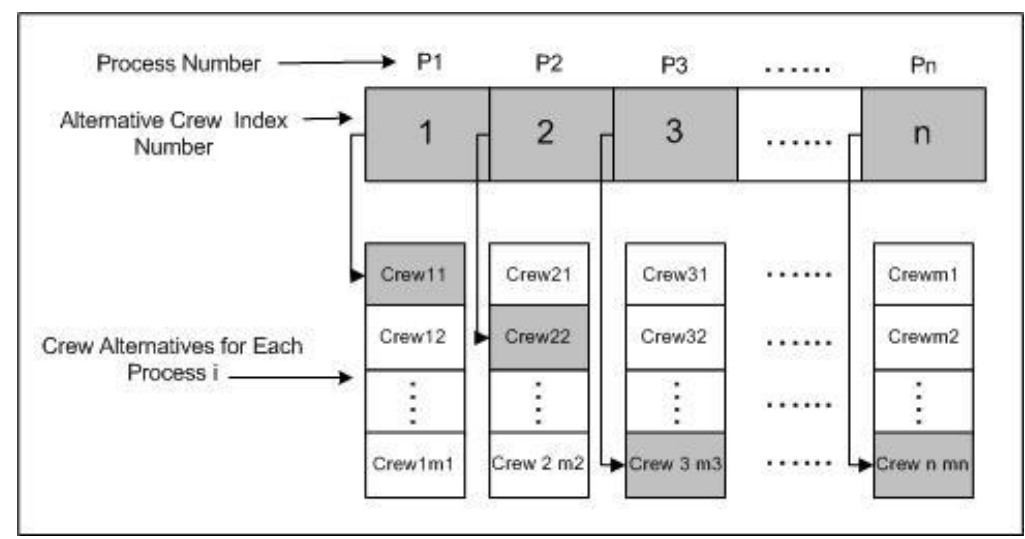

Figure 5: chromosome representation for the crew allocation problem

In figure 5, each integer number of each gene would give the crew index number of the set of crew alternatives associated with that gene, i.e; this number would give the index of a crew that could be used in the solution as each gene has different possible alternatives of crews. A chromosome is encoded in a decimal way with the chromosome length is representing the maximum number of processes involved in any labor-driven production facility.

The decision variables are the number of sets of crews available to allocate to each process. To evaluate each chromosome, a single objective function has been identified and adopted to minimize labor costs. Many restrictions can be determined which limit production amounts, alternative crews, and operational hours (shifts). In some situations, a trade-off exists between idle time and cost (El-Rayes et. al., 2001). In this study, only direct cost is considered as a substantial cost and GAs have been used productively in a number of studies in order to optimize costs (Kim and Adeli 2001, Sarma and Adeli 2002). 


\section{Al-Bazi \& Dawood}

The objective function is applied to evaluate total resource costs from information identified in previous job-shop cost records. The equation used to calculate the objective function is:

$$
f \varangle_{i}=\sum_{i=1}^{n} B R C_{i}+I R C_{i}+R C P U_{i}
$$

Where:

$\mathrm{n}$ is the number of labor-driven processes

$B R C_{i}$ : incurred cost per hour when using a laborer for set of solution i.

$I R C_{i}$ : incurred cost per hour when laborer is idle for set of solution $\mathrm{i}$ and

$R C P U_{i}$ : incurred cost per use of fixed or physical resource for set of solution $\mathrm{i}$; senior skilled bonus can be considered in such cost.

As an initial starting point, a population of solutions (chromosomes) is generated randomly using Monte-Carlo sampling. Population size and other GA operators' parameters influence the solution and simulation time (Boyabatli 2007). As one of the random sampling techniques, Monte Carlo is used to select crew alternative for each gene (i.e process). This generates a random number for each gene which is used to select the alternative crews for each process. The range of random numbers for each gene can be determined using the following constraint

$$
\operatorname{MinCA}_{i} \leq R_{i} \leq \operatorname{Max} C A_{i}
$$

where

$R_{i}$ is an integer random number for each chromosome $\mathrm{i}$

$\operatorname{MinCA}_{i}$ is minimum number of crews alternative in gene $\mathrm{i}$ and

$\operatorname{MaxCA}_{i}$ is maximum number of crews alternative in gene $\mathrm{i}$

In the proposed model, the user is given the possibility to input the population size. Once the population is generated, each chromosome is evaluated individually using the developed simulation model. GA operators are tailored to suit this type of allocation problem. Next, "Class Interval" selection, "Dynamic Crossover" and "Mutation" Strategies are explained. 


\section{The Proposed "Class Interval" Selection Strategy (CISS)}

The promising chromosomes with least costs will be given a higher chance of selection, and are named the "class interval" strategy, which is based on the concept used in the descriptive statistics. (Healey, 2009)

The top of the minimum costs chromosome will be given higher weighting than others by calculating the fitness function according to equation (2):

$$
G x_{i}=\operatorname{Max}-f \mathbf{Q}_{i}^{-}
$$

where

$G x_{i}$ : Fitness function of chromosome $\mathrm{i}$

$f \boldsymbol{C}_{i}$ : Sorted objective function for top promising chromosomes and

Max: The largest cost value in the best promising chromosomes

Repetition of the generated chromosome is not allowed, meaning that all generated chromosomes should be unique over the evolution process. To construct the chromosome's class interval, a relative fitness function $R G x_{i}$ is calculated using equation 3:

$$
R G x_{i}=\frac{G x_{i}}{\sum_{i=1}^{m} G x_{i}} \quad, \mathrm{i}=1, . . m
$$

where

$R G x_{i}:$ Relative fitness function of chromosome $\mathrm{i}$ and $m:$ is the population size

The cumulative relative fitness function is then calculated, as it is useful to determine the desired class width using equation 4 :

$$
C R G x_{i}=C R G x_{i-1}+R G x_{i} \quad, \mathrm{i}>\quad 1
$$

where

$$
C R G x_{1}=G x_{1}
$$

$R G x_{i}:$ Relative fitness function of chromosome $\mathrm{i}$ and $C R G x_{i}$ : Cumulative Relative fitness function of chromosome $\mathrm{i}$ 
Subsequently, the possible interval of occurance for each chromosome is determined from the class intervals. The interval associated for each chromosome will represent the chance range of that chromosome to be selected by any generated (0-1) random variate as in figure 6 :



Figure 6: the proposed "class-interval” selection rule

In the proposed selection strategy and after sorting the resulting costs obtained from equation 1 in ascending order, the chromosomes with minimum costs are selected from the augmented population pool as the promising pair of chromosomes. The fitness function is then calculated using equation 2 , and the result is sorted in descending order for a better selection at a later stage. The relative fitness function is calculated using equation 3 as a requirement for constructing the chromosomes class interval. Cumulative relative frequency is then calculated using equation 4 as the upper bound of the chromosome's opportunity to be selected. The lower boundary can be identified to represent the possible range of each chromosome to be selected. This strategy is derived from the class interval concept in descriptive statistics and has been found to be useful for 
chromosomes selection. The idea of modifying this concept is to give a greater opportunity for the promising chromosomes to be selected again. The chromosomes with minimum cost or a higher fitness function will be given a wider range of interval so random variants will be more likely to lie within this range.

\section{Dynamic Points Crossover (DPC)}

The crossover operation in a conventional GA is based on the exchange of genes between two fixed length chromosomes when string coding is applied (Zhang et. al., 2005). In the "SIM_Crew" model, DPC is developed to achieve the best random exchange of genes between each pair of chromosomes, as shown in figure 7:



\begin{tabular}{|c|c|c|c|c|c|c|c|c|c|}
\hline-1 & 2 & 3 & 4 & 5 & $6_{x}$ & 7 & 8 & 9 & \\
\hline P1Crew1 & P2Crew1 & P3Crew3 & P4Crew1 & P5Crew3 & P6Crew3 & P7Crew1 & P8Crew1 & P9Crew1 & P10Crew1 \\
\hline P1Crew3 & P2Crew3 & P3Crew1 & P4Crew2 & P5Crew4 & P6Crew2 & P7Crew2 & P8Crew3 & P9Crew3 & P10Crew2 \\
\hline
\end{tabular}

Figure 7: the Dynamic-Points Crossover (DPC) strategy

In this type of crossover, random numbers of size (n) are generated to be associated with each gene; a vertical crossover takes place to exchange or alternate subsequently $\mathrm{n}$ gene(s) of the first chromosome with the opposite gene of the second selected chromosome after satisfying the condition below: 
If the probability of crossing over a gene $\leq$ the random number associated with that gene, then the crossing over of that gene is possible.

For example, suppose five random numbers satisfy the swapping condition at genes 2,4 , and 4 , the chosen places or genes will be exchanged between each selected pair of chromosomes.

\section{Dynamic-Points Mutation (DPM)}

This mutation operator is similar to the developed crossover operator where random numbers of size (m) are generated to be attached to each gene. The selected genes are then specified for further mutation. The possible crew place to carry out a particular process is then generated randomly for each chosen gene. The mutation process continues until a unique chromosome is satisfied to be placed into the new population for further testing is shown in figure 8 .
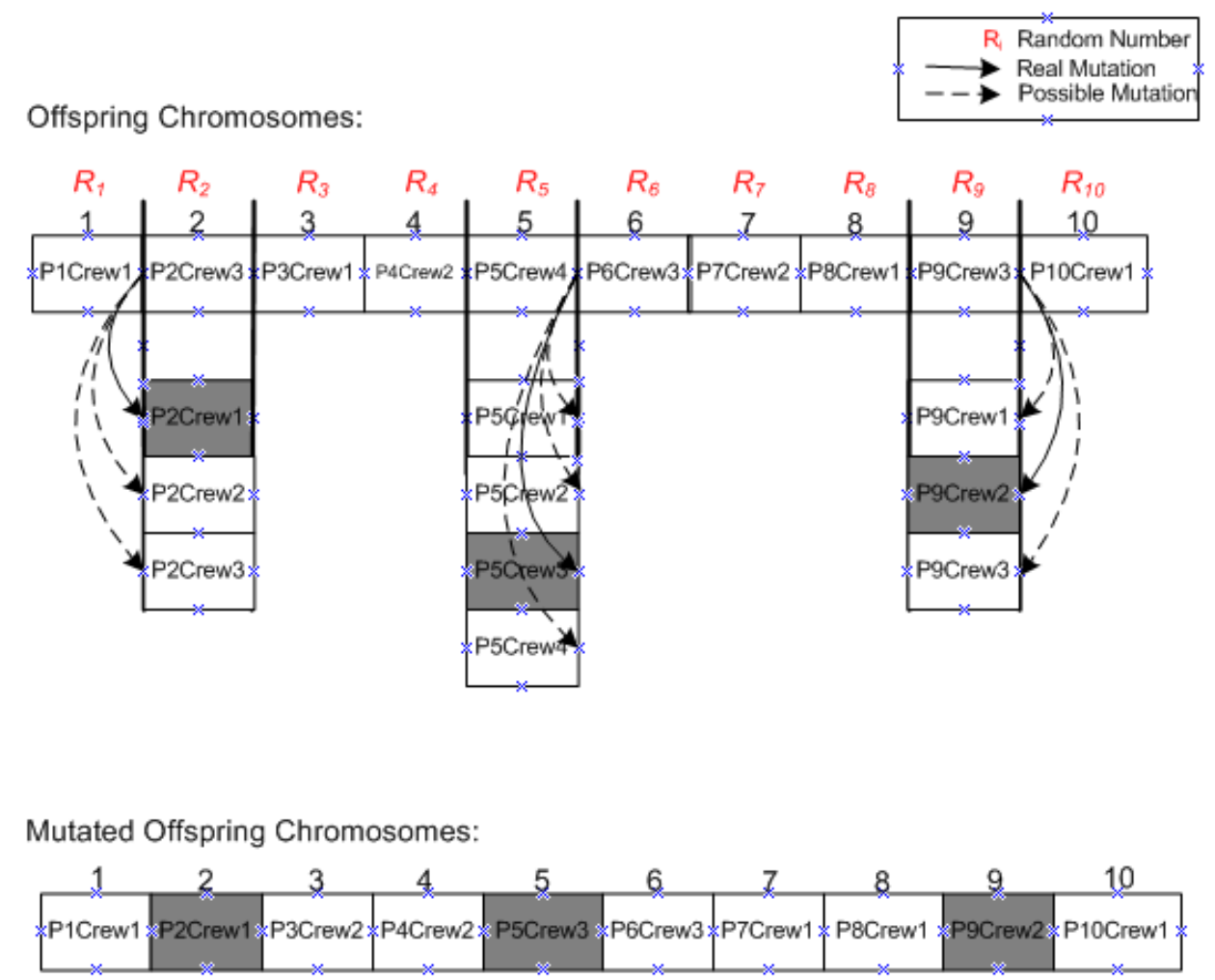

Figure 8 the Dynamic Points Mutation (DPM) strategy

After a crossover operator has taken place, each chromosome is then mutated individually. The same strategy of the Dynamic Crossover can be individually applied to each chromosome and the 


\section{Al-Bazi \& Dawood}

same exchange condition can be used to mutate genes. The selected genes can be mutated with its pool "list of alternative crews" using the Monte-Carlo simulation.

\section{Avoiding of Chromosome Duplication}

As solutions (chromosomes) are generated randomly using the GAs operators, the possibility of repeated solution exists. Such repetition causes additional calculations and subsequently leads to an increase in the calculation time. In order to avoid duplication of chromosomes during evaluation and to minimize evaluation time and calculation operations, "one search strategy" is developed to search for any duplications and to discard any repeated chromosomes. This enables the "SIM_Crew" engine to evaluate just non-duplicated chromosomes for a faster and better investigation.

The "one search strategy" enables investigation of the current generation chromosome to check whether any chromosome is repeated in the chromosome list which is stored in access database. The non-repeated chromosomes can be stored temporarily in the chromosomes access database under the (-1) generation number. Defining chromosomes with the (-1) generation number for the non-duplicate chromosomes eases the discarding of them after copying them to the VBA database. Figure 9 below shows the "one-search strategy" steps that "SIM_Crew" applies to filter the resulted chromosomes. 




Figure 9: “one search strategy" flowchart for duplication

In this developed flowchart, "one search strategy" is developed to save computation time and program codes. In one search strategy and after selecting a pair of chromosomes, crossover will take place by exchanging selected genes using the probabilistic dynamic strategy. To add more randomness to the crossed-over chromosomes, each chromosome should be mutated individually with its available list of alternatives for each process using the Monte Carlo (MC) sampling method. The mutated chromosome will be checked with Access database which involves all chromosomes generated for all previous populations (population history), then, if the tested chromosome is duplicated, the mutation process should continue until a unique chromosome is obtained.

After finding a unique chromosome, a copy of this chromosome should be sent to access database population list under the (-1) generation number and another copy to the VBA array which 


\section{Al-Bazi \& Dawood}

involves all unique chromosomes for the current generation for evaluation purpose. After testing all chromosomes for duplication, all chromosomes having the (-1) generation number should be discarded from the access database population history and the VBA array should be prepared to be fed to the simulation engine for evaluation purposes.

The purpose of the temporary chromosome is to assist checking for duplicated entries while the next generation is being created. As each chromosome is generated through the crossover mutation operators, it is stored into the database with a generation number of (-1) a flag that eases the deletion of non-repeated chromosomes. This means that all past, current and proposed chromosomes are held in one location, so a single search can check if the new chromosome is a duplicate.

The "one search strategy" ensures that all chromosomes are unique before evaluating them; therefore, there is no point to test for repeated chromosomes as it wastes time and computational efforts.

\section{The Case Study}

A case study of one of the largest 'sleepers' products precast concrete industry in the UK was developed. In this sleeper manufacturing system, the production process involves using a wide range of different resources, including labor, equipment, and materials. For planning and control purposes, precast operations are broken down into activities/tasks, so that resources can be economically allocated and controlled.

The railway sleeper concrete manufacturing system is investigated in this study and is divided into two main production sections; each having two labor-intensive production lines and shared resources (labor and machines) are used to carry out job/activity on each production line alternately. Eight processes including curing are applied to the sleeper.

Two types of multi-skilled workers were used to carry out jobs in the two production lines: charge workers (multi-skilled workers in charge of operators) and operators (multi-skilled 


\section{Al-Bazi \& Dawood}

workers). Each multi-skilled worker has the skills to carry out a number of possible activities, depending on the accumulated skills of the worker and his/her ability to work on more than one process.

In production section 2, eleven operators and two charge workers are used to carry out tasks, and thirteen operators and four charge hands in production section 3. On any of the production lines, a "reusable mold" is the main resource, which consists of a gang of molds that can be used to produce either the same or different types of sleeper. A wide range of sleepers are produced by the production processes presented in figure 2 such as the 'G44', '5EF28', 'EG47', and 'EG47 3R'. The logic of these production processes was presented by Dawood and Al-Bazi (2009).

Resources assigned to each mold are shared resources (machines, laborers) which are applied to produce the finished sleeper. The job-shop layout consists of three zones: materials zone, concrete mix zone, and production zone. In the material zone, all steel wire rolls, plastic spacers, pandrols, and other finishing accessories are stored nearby to be available when needed. A number of mechanical resources can be stored in this area after using them.

After a detailed investigation of each process in the company, a simulation model was developed using ARENA simulation software to simulate the production processes of the sleeper concrete manufacturing system. All relevant data were collected by conducting onsite visits, using flowcharts and process maps, interviews and the stop watch technique as relevant data collection techniques. The crew alternatives for each production process were identified by the production planner according to the accumulated experience record of a worker as shown in table 1.

Table 1: number of crew alternatives for each process 


\begin{tabular}{|c|c|c|c|c|c|c|c|}
\hline 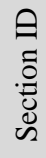 & 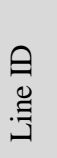 & Process ID & $\begin{array}{l}\text { Number of } \\
\text { Crew } \\
\text { Alternatives }\end{array}$ & 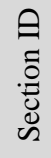 & $\stackrel{\overbrace{}}{\ominus}$ & Process ID & $\begin{array}{l}\text { Number of } \\
\text { Crew } \\
\text { Alternatives }\end{array}$ \\
\hline \multirow{14}{*}{ 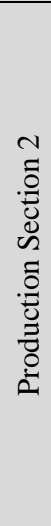 } & \multirow{7}{*}{ 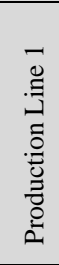 } & Setup & 4 & \multirow{14}{*}{ 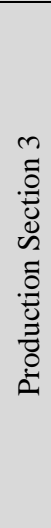 } & \multirow{7}{*}{ 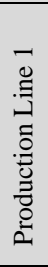 } & Setup & 3 \\
\hline & & Run Strand & 4 & & & Run Strand & 3 \\
\hline & & Stress & 4 & & & Stress & 3 \\
\hline & & Casting & 4 & & & Casting & 3 \\
\hline & & Sawoff & 3 & & & Sawoff & 3 \\
\hline & & Demolding & 4 & & & Demolding & 3 \\
\hline & & Finishing & 4 & & & Finishing & 3 \\
\hline & \multirow{7}{*}{ 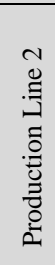 } & Setup & 4 & & \multirow{7}{*}{ 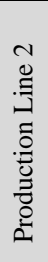 } & Setup & 3 \\
\hline & & Run Strand & 4 & & & Run Strand & 3 \\
\hline & & Stress & 4 & & & Stress & 3 \\
\hline & & Casting & 4 & & & Casting & 3 \\
\hline & & Sawoff & 3 & & & Sawoff & 3 \\
\hline & & Demolding & 4 & & & Demolding & 3 \\
\hline & & Finishing & 4 & & & Finishing & 3 \\
\hline
\end{tabular}

According to the above table, the possible number of allocation plan is equal to $722,204,136$ million which demonstrate the crew allocation problem being investigated to be a combinatorially complex one. However, more detail about crew formation data and worker details are confidential and cannot be published in this study.

Twenty-eight production processes divided between four production lines in two production sections (seven repetitive processes at each) were considered in the $3 \mathrm{D}$ simulation model. The 3D simulation model can be used as a communication tool to convey the idea of how a sleeper production system works and how it performs to avoid costly mistakes as early as possible. (Anttila, 2005). The purpose of visualization was to verify the simulation model and to provide production managers with more advanced means of communication. However, the 3D animation was enough to verify the logic used in constructing the simulation model. See figure 10. 


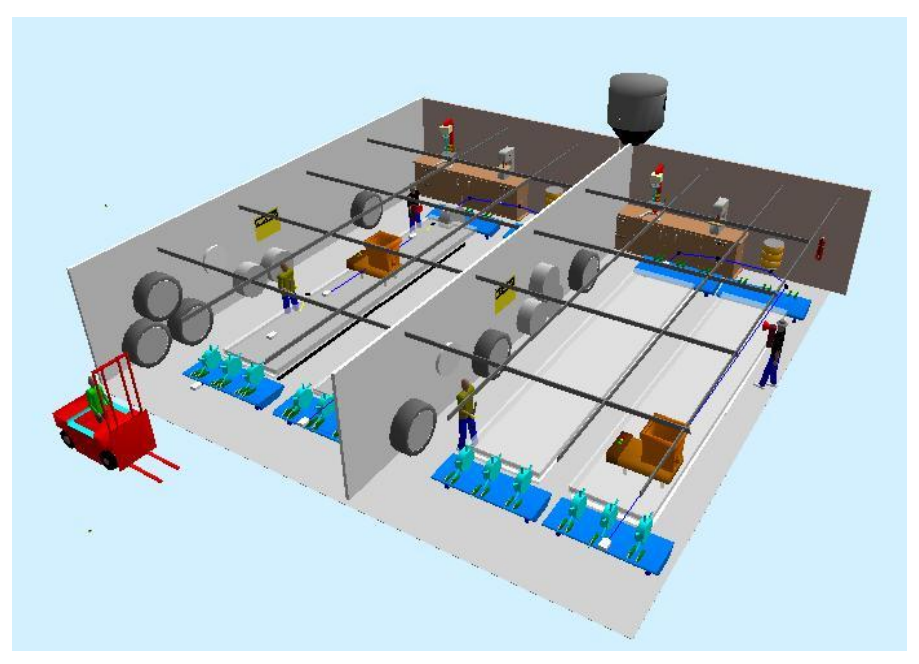

Figure 10: depicts visualization of the sleepers manufacturing system

A set of crews specified to carry out each production process which had been suggested by the production manager were fed into the system database. Class Interval Selection Strategy (CISS) was used as a selection strategy, while Dynamic Points Crossover (DPC) and Dynamic Points Mutation (DPM) were chosen as a crossover and mutation strategy. Fourty generations with a population of size 20 was used to obtain a stable result. In order to analyze opportunites for improvement in the sleeper precast concrete manufacturing system, an optimization study will be conducted in the next section to identify the impact of allocation plans on system performance.

\subsection{Running Experimental Work}

The objective of this study was to iteratively generate and test possible crew allocation scenarios which can be evaluated using simulation until the minimum resource allocation cost is found. The GAs module is used to iteratively generate and evolve allocation scenarios. The simulation module is then utilized to test the impact of each allocation scenario on the performance of the manufacturing system in terms of resource utilization and costs.

In most cases, the algorithm converges after 25 generations which takes about 7-8 hours and requires a memory of $512 \mathrm{MB}$. The population size is set to be 20 chromosomes. The chromosomes size is defined to be equal to the number of processes. The probability of crossing over a gene and mutating a gene is identified to be $0.2,0.3$ respectively (several sets of different 
probabilities have been attempted without any significant effects). The probability of mutation is higher than crossover, as the mutation operator in this system plays a vital role in mutating repeated chromosomes in order to obtain the unique one.

For the model settings above, a simulation run has been performed to check the functionality of the proposed system and determine which type of development the model can add to reduced costs.

\subsection{Analysis and Interpretation}

The results of the case study are shown in figures 11-15



Figure 11: costs reduction using “SIM_Crew” allocation System

Figure 11 shows that a significant drop in cost takes place after the 2nd generation with slight cost reductions in a number of generations. The GA dynamic probabilistic operators have successfully explored more promising solution areas on the mentioned generations. Allocation cost tends to have no improvement after 25 generations. This best scenario drove the allocation cost equal to $£ 48300$ in which a return of $1.5 \%$ (about $£ 300$ per five working days) is achieved.

To verify that the GA converges to the optimal solution, we applied the average results to test the improvement and convergence of the solutions, as in figure 12. 


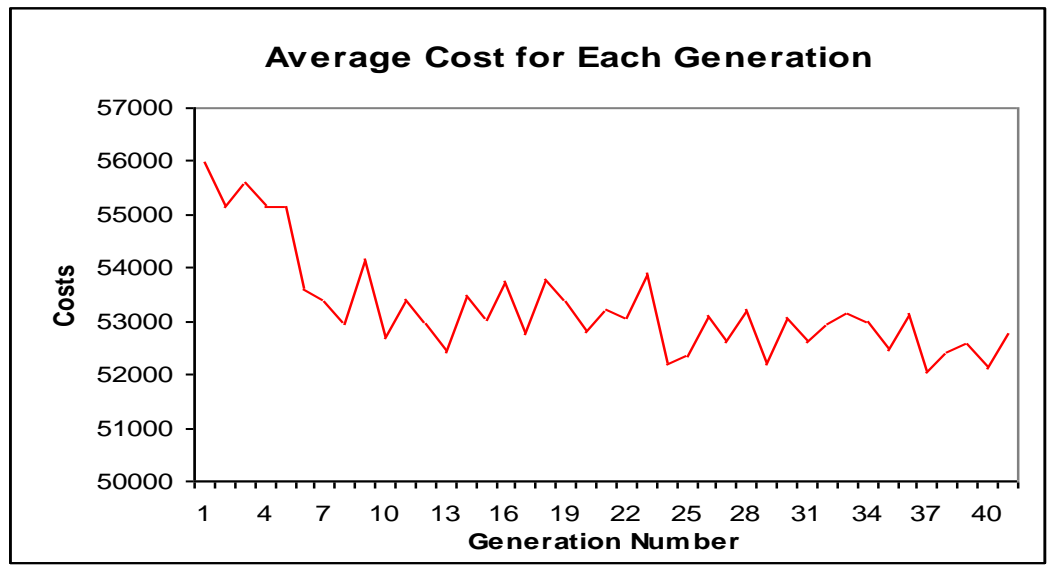

Figure 12: reduction trend in costs using "SIM_Crew" System

This figure shows improvement of the solutions over generations: generation by generation the average solution is improved due to the evolving process available in the algorithm used. The utilization of fixed resources and labor is optimized in order to identify the optimal utilization that could minimize the total allocation cost. A sample of two workers from different skills backgrounds working with a concrete mold has been selected from 47 resources to be presented in this paper. The best chromosome of each generation is selected to present the optimized utilization of resources of that chromosome as shown in figures 13-15:

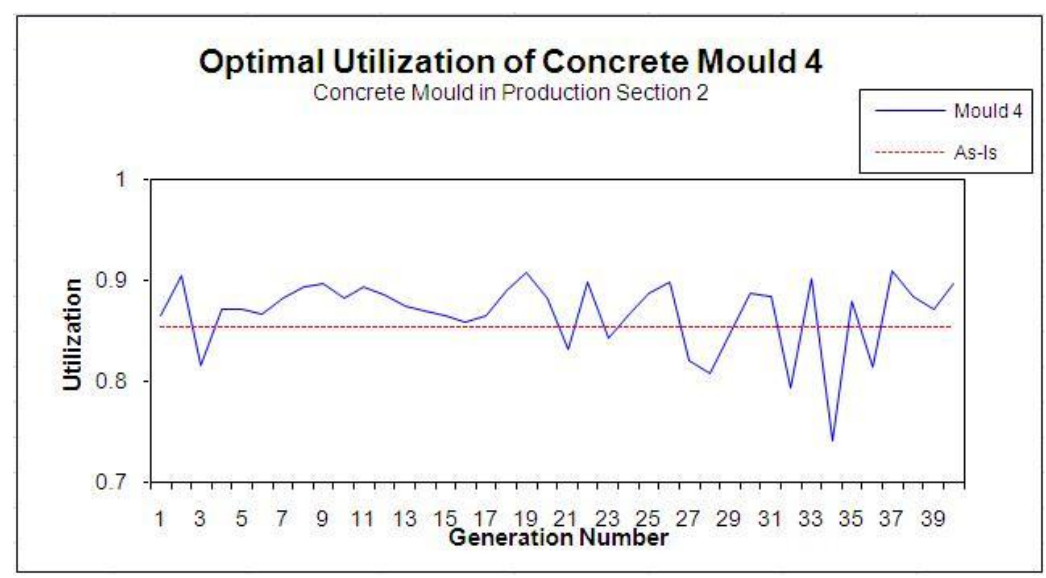

Figure 13: mold utilization optimization using "SIM_Crew"

In figure 13, the utilization of the used concrete mold at section 3 is optimized to determine the required level of utilization needed for the mold resource to minimize the total production costs. Fixed resources such as the mold have shown a highly stable utilization because of their 
continuous use. For laborer resources, one of the operative's utilization profiles is shown in figure 14.



Figure 14: operative labor utilization optimization using "SIM_Crew"

In figure 14, the utilization of worker 21 who is an operative laborer working in section 3 is optimized to determine the required level of utilization needed to minimize the total manufacturing costs. However, operators have a higher utilization as they are involved in carrying out most of the manual work. Skilled worker utilization is also calculated as shown in figure 15 .

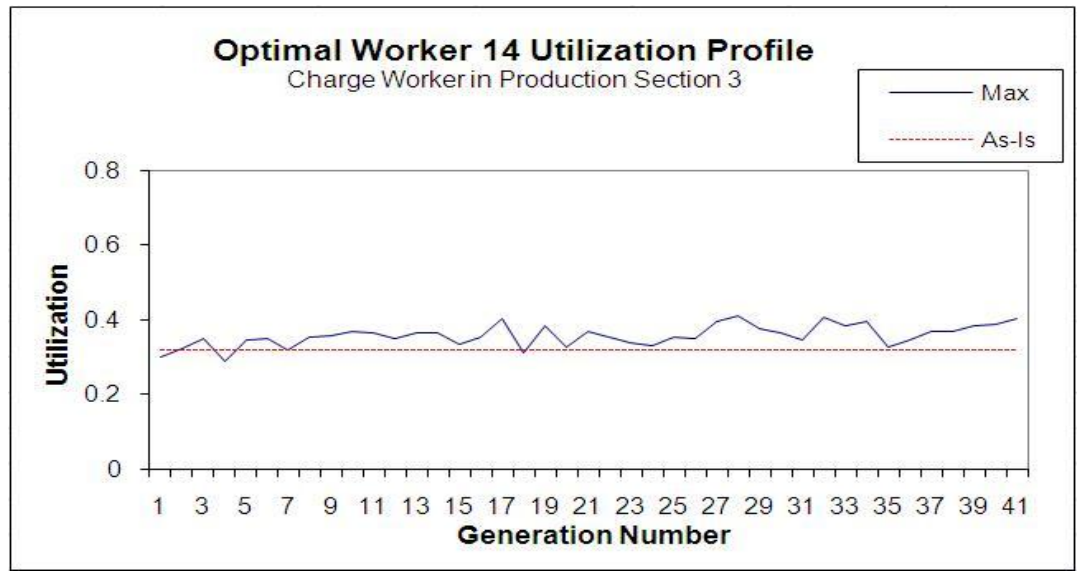

Figure 15: skilled labor utilization optimization using "SIM_Crew"

In figure 15 , the utilization of worker 14 , a senior charge worker working in section 3 is optimized to determine the required level of utilization to minimize the total allocation cost. 


\section{Al-Bazi \& Dawood}

Skilled workers are less utilized as their main duty is supervision and guidance. The resources utilization charts presented in figures 14-15 reveal that utilization of shared the laborer fluctuates as the objective function seeks to achieve the required resources utilization. The non-shared resources such as molds have a stable utilization as they are used only by one set of workers at a time.

Different levels of utilization of each selected resource are shown through many generations, so the fluctuation of shared resource utilization is remedied by varying the utilization of other resources to satisfy the desired objective function. The last generation will show the optimized utilization required to achieve minimum total production costs.

The developed simulation model has not considered other factors as breakdown or mixer problems. In addition, none of the environmental impacts such as weather have been considered in this study. These shortcomings can be addressed in future research projects. To summarize the key points of this work, the next section will be devoted to present this summary. In addition, future work will be suggested for potential improvement.

\section{Conclusions and Future Works}

In this paper, a GA-based simulation model to solve the crew allocation problem has been developed. The proposed algorithm was tested in a real life case study and the results were compared with the current operating strategy. In addition, a newly developed selection strategy named "Class Interval" was also applied to evaluate the efficiency of the proposed algorithm in selecting promising chromosomes.

It has been concluded that the utilization factor has a significant impact on crew allocation cost through the effect of the idle time factor on the allocation cost. Optimal usage of resources (labor/machines) in conjunction with a minimized production time can lead to a minimum allocation cost. 


\section{Al-Bazi \& Dawood}

As further developments, the GA-based simulation algorithm may be suitably modified and employed to solve more complicated crew allocation problems by considering the detailed modeling of processes with more than one working shift. More factors such as breakdown and environmental impact will be considered in more detail while developing the allocation system. The Monte Carlo Simulation can be used to assign a random crew to each process. This type of heuristic rules can be compared with the results of GAs to check the efficiency of the proposed allocation strategy.

\section{References}

Adeli, H. and Cheng, N.-T. (1994a), "Augmented Lagrangian Genetic Algorithm for Structural Optimization", Journal of Aerospace Engineering, ASCE, Vol. 7, No. 1, pp. 104-118.

Adeli, H. and Cheng, N.-T. (1994b), "Concurrent Genetic Algorithms for Optimization of Large Structures", Journal of Aerospace Engineering, ASCE, Vol. 7, No. 3, pp. 276-296

Adeli, H. and Karim, A. (1997). Scheduling/Cost Optimization and Neural Dynamics Model for Construction. Journal of Construction Management and Engineering, ASCE, Volume 123, Number 4, pp. 450-458.

Adeli, H. and Kumar, S. (1995a), "Distributed Genetic Algorithms for Structural Optimization", Journal of Aerospace Engineering, Vol. 8, No. 3, pp. 156-163.

Adeli, H. and Kumar, S. (1995b), "Concurrent Structural Optimization on a Massively Parallel Supercomputer", Journal of Structural Engineering, ASCE, Vol. 121, No. 11, pp. 1588-1597.

Adeli, H. and Wu, M. (1998), Regularization Neural Network for Construction Cost Estimation. Journal of Construction Engineering and Management, ASCE, Volume 124, Number 1, pp. 1824.

Adeli, H. and Hung, S. L. (1995). Machine learning-neural networks, genetic algorithms, and fuzzy systems. Wiley, New York. 
Adeli, H. and Park, H. S. (1996), Hybird CPN-Neural dynamics model for discrete optimization of steel structures. Microcomputers in Civil Engineering, 11(5), 335-366.

Alcaraz, J., and Maroto C. (2001). A robust genetic algorithm for resource allocation in project scheduling. Annals of operations research, Volume 102, Number 1-4, February, pp. 83-109

Anttila, M. (2005). 3D manufacturing simulation-improving the return on investment. Proceedings 19th European Conference on Modeling and Simulation.

Boyabatli, O. (2007). Parameter selection in genetic algorithms. Journal of Systemic, Cybernetics and information (JSCI), Volume 2, Number 4, pp. 78-83

Carro-Calvo, L., Salcedo-Sanz, S., Gil-Pita, R., Portilla-Figueras, A., and Rosa-Zurera, M. (2009), An Evolutive Multiclass Algorithm for Automatic Classification of High Range Resolution Radar Targets,” Integrated Computer-Aided Engineering, 16:1, pp. 51-60.

Cheng, T.M. and Yan, R.Z. (2009), "Integrating Messy Genetic Algorithms and Simulation to Optimize Resource Utilization, Computer-Aided Civil and Infrastructure Engineering, 24:6, pp. 401-415.

Chi, S., Caldas, C.H., and Kim, D.Y. (2009), "A Methodology for Object Identification and Tracking in Construction based on Spatial Modeling and Image Matching Techniques," Computer-Aided Civil and Infrastructure Engineering, 24:3, pp. 199-211.

Dawood, N, Ahmed, R. and Dean, J. (2007) "Modeling of Precast Concrete Production Operations and Innovations: A Simulation Approach”. Manubuild Conference, Rotterdam, 25-26 April.

Dawood, N. and Al-Bazi, A. (2009) "Using Genetic Algorithms to Improve Crew Allocation Process in Labour-Intensive Industries". The 2009 ASCE Workshop on Computing in Civil Engineering, Texas, Austin, p166-175.

El-Rayes , K., and Moselhi, O. (2001). Optimizing resource utilization for repetitive construction projects. Journal of Construction Engineering and Management, Vol. 127, No.1, pp. 18-27 
Glover, F., Kelly, J.P., and Laguna, M. (1999), New advances for wedding optimization and simulation, in Proceedings of the 1999Winter Simulation Conference, 255-260.

Healey, F.J. (2009), Statistics: Tool for social research, (8th ed.), Belmont, CA: Wadsworth Cengage Learning, USA.

Hegazy, T., ASCE, M. and Kassab, M. (2003). Resource optimization using combined simulation and genetic algorithms. Journal of Construction Engineering and Management, Volume 129, Issue 6, pp. 698-705

Hung S.L. and Adeli, H. (1994), "A Parallel Genetic/Neural Network Learning Algorithm for MIMD Shared Memory Machines", IEEE Transactions on Neural Networks, Vol. 5, No. 6, pp. 900-909.

Ipsilandis, P.G. (2006). Multi-objective optimization in linear repetitive project scheduling. Operational Research. An International Journal. Volume 6, Number 3, pp.255-269

Jiang, X. and Adeli, H. (2008), "Neuro-Genetic Algorithm for Nonlinear Active Control of Highrise Buildings, International Journal for Numerical Methods in Engineering, Vol. 75, No. 8, pp. 770-786.

Kang, M.W., Schonfeld, P., and Yang, N. (2009), Prescreening and Repairing in a Genetic Algorithm for Highway Alignment Optimization," Computer-Aided Civil and Infrastructure Engineering, 24:2, pp. 109-119.

Kim, H. and Adeli, H. (2001), "Discrete cost optimization of composite floors using a floating point genetic algorithm", Engineering Optimization, Vol. 33, No. 4, pp. 485-501.

Kumar, A. J. S., Arunadevi, J., Mohan, V. (2009). Intelligent Transport Route Planning Using Genetic Algorithms in Path Computation Algorithms. European Journal of Scientific Research. Volume 25, Number 3, pp.463-468 


\section{Al-Bazi \& Dawood}

Lee, H., Kim, E., and Park, M. (2007), "A genetic feature weighting scheme for pattern recognition," Integrated Computer-Aided Engineering, 14:2, pp. 161-171.

Li, H., Love, P., and Ogunlana, S. (1998). Genetic algorithum compared to nonlinear optimization for labor and equipment assignment. Building Research and Information, Volume 26, Number 6, pp.322-329

Lu, M., Lam, Hoi-Ching, Tang S.L. (2008). Skilled laborer management in handling concurrent building projects at multiple sites: the bar benders job scheduling problem. First International Conference on Construction in Developing Countries (ICCIDC-I), August 4-5, Karachi, Pakistan Marzouk, M. Hisham Zein El-Dein, and Moheeb El-Said. (2007). Application of Computer Simulation to Construction of Incremental Launching Bridges. Journal of Civil Engineering and Management, Volume XIII, Number 1, pp. 27-36.

Marzouk , M., and Moselhi, O. (2004). Multi-objective optimization of earthmoving operations. Journal of Construction Engineering and Management, Vol. 130, No.1, pp. 105-113

Mathakari, S., Gardoni, P., Agarwal, P., Raich, A., and Haukaas, T. (2007), "Reliability-based Optimal Design of Electrical Transmission Towers Using Multi-objective Genetic Algorithms,“ Computer-Aided Civil and Infrastructure Engineering, 22:4, pp. 282-292.

Moselhi, O., Alshibani, A. (2007), Crew optimization in planning and control of earthmoving operations using spatial technologies, Journal of Information Technology in Construction, The International Council for Research and Innovation in Building and Construction, available at: http://www.itcon.org/cgi-bin/works/Show?2007_7 (accessed 23 February 2009), Vol. 12 pp.12137.

Nassar, K. (2005). Evolutionary optimization of resource allocation in repetitive construction schedules. Journal of Information Technology in Construction (ITcon), Volume 10, pp.265-273 Rekapalli, P.V., Martínez, J.C., and Kamat, V.R. (2009), "Algorithms for Accurate ThreeDimensional Scene Graph Updates in High Speed Animations of Simulated Construction Operations," Computer-Aided Civil and Infrastructure Engineering, 24:3, pp. 186-198. 
Smith, J.F. and ThanhVu H. Nguyen, T.H. (2007), “Autonomous and cooperative robotic behavior based on fuzzy logic and genetic programming," Integrated Computer-Aided Engineering, 14:2, pp. 141-159.

Sarma, K. and Adeli, H. (2000a), "Fuzzy Genetic Algorithm for Optimization of Steel Structures", Journal of Structural Engineering, ASCE, Vol. 126, No. 5, pp. 596-604.

Sarma, K. and Adeli, H. (2000b), "Fuzzy Discrete Multicriteria Cost Optimization of Steel Structures", Journal of Structural Engineering, ASCE, Vol. 126, No. 11, pp. 13391347.

Sarma, K.C. and Adeli, H. (2001), "Bi-Level Parallel Genetic Algorithms for Optimization of Large Steel Structures", Computer-Aided Civil and Infrastructure Engineering, 16 (5), pp. 295-304.

Sarma, K.C. and Adeli, H. (2002), "Life-Cycle Cost Optimization of Steel Structures", International Journal for Numerical Methods in Engineering, Vol. 55, No. 12, pp. 1451-1462.

Senouci, A.B. and Adeli, H. (2001). Resource Scheduling using Neural Dynamics Model of Adeli and Park. Journal of Construction Engineering and Management, ASCE, Volume 127, Number 1, pp. 28-34.

Shi, L. (2000). A new algorithm for stochastic discrete allocation optimization. Journal of discrete Event Dynamic Systems, Volume 10, Number 3, pp.271-294

Teklu, F. Sumalee, A., and Watling, D. (2007), "A Genetic Algorithm for Optimizing Traffic Control Signals Considering Routing," Computer-Aided Civil and Infrastructure Engineering, 22:1, pp. 31-43.

Vlahogianni, E.I., Karlaftis, M.G., and Golias, J.C. (2007) Spatio-Temporal Short-Term Urban Traffic Flow Forecasting Using Genetically-Optimized Modular Networks," Computer-Aided Civil and Infrastructure Engineering, Vol. 22, No. 5, pp. 317-325. 


\section{Al-Bazi \& Dawood}

Watkins, M. Mukherjee, A., Onder, N. and Mattila K. G. (2007). Understanding Labor Productivity as an Emergent Property of Individual and Crew Interactions on a Construction Site. Proceedings IGLC-15, July, Michigan, USA.

Zhang H., Li H. (2004). Simulation-based optimization for dynamic resource allocation. Journal of Automation in Construction Volume 13, pp. 409-420.

Zhang X. and Armstrong, M.P. (2005). Using a genetic algorithm to generate alternatives for multi-objective corridor location problems. Proceedings of the 8th International GeoComputation Conference. 